\title{
Anti-apoptotic effects of pan-caspase inhibitor (Z-VAD), SOD or catalase on antimycin A-induced HeLa cell death
}

\author{
YONG HWAN HAN, SUHN HEE KIM, SUNG ZOO KIM and WOO HYUN PARK
}

\author{
Department of Physiology, Medical School, Institute for Medical Sciences, Center for Healthcare \\ Technology Development, Chonbuk National University, JeonJu 561-180, Korea
}

Received June 18, 2008; Accepted January 5, 2009

DOI: 10.3892/mmr_00000101

\begin{abstract}
Antimycin A (AMA) is an inhibitor of the electron transport chain in mitochondria. In this study, we investigated the anti-apoptotic effects of pan-caspase inhibitor (Z-VAD), superoxide dismutase (SOD) or catalase on AMA-induced HeLa cell death in relation to the cell cycle. Treatment with Z-VAD, SOD or catalase rescued some HeLa cells from AMA-induced apoptosis, but did not prevent the growth inhibition of HeLa cells by AMA. DNA flow cytometric analysis indicated that treatment with AMA significantly induced an S-phase arrest of the cell cycle at $72 \mathrm{~h}$. Interestingly, Z-VAD, SOD and catalase intensified S-phase arrest in AMA-treated cells. In conclusion, treatment with Z-VAD, SOD or catalase decreased apoptotic levels in AMA-treated cells, which was associated with the enhancement of the S-phase arrest of the cell cycle in these cells.
\end{abstract}

\section{Introduction}

Antimycin A (AMA) is a mixture primarily composed of antimycin A1 and A3 derived from Streptomyces kitazawensis (1). AMA inhibits succinate and NADH oxidase, and specifically inhibits mitochondrial electron transport between cytochromes b and c (2-4). Inhibition of electron transport causes a collapse of the proton gradient across the mitochondrial inner membrane, thereby collapsing mitochondrial membrane potential $\left(\Delta \Psi_{\mathrm{m}}\right)(2,4,5)$. This inhibition can produce reactive oxygen species (ROS) $(5,6)$, which include hydrogen peroxide

Correspondence to: Dr Woo Hyun Park, Department of Physiology, Medical School, Chonbuk National University, JeonJu 561-180, Korea

E-mail: parkwh71@chonbuk.ac.kr

Abbreviations: AMA, antimycin A; ROS, reactive oxygen species; NADPH, nicotine adenine diphosphate; XO, xanthine oxidase; SOD, superoxide dismutase; FBS, fetal bovine serum; PBS, phosphate buffer saline; FITC, fluorescein isothiocyanate; MTT, 3(4,5-dimethylthiazol-2-yl)-2,5-diphenyltetrazolium bromide; PI, propidium iodide; CDKI, cyclin-dependent kinase inhibitor; Z-VAD-FMK, benzyloxycarbonyl-Val-Ala-Asp-fluoromethylketone

Key words: antimycin A, reactive oxygen species, Z-VAD, superoxide dismutase, catalase, apoptosis, cell cycle
$\left(\mathrm{H}_{2} \mathrm{O}_{2}\right)$, superoxide anion $\left(\mathrm{O}_{2}{ }^{-}\right)$, hydroxyl radical $\left({ }^{\circ} \mathrm{OH}\right)$ and peroxynitrite (ONOO-). A change in the redox state of the tissue implies a change in ROS generation or metabolism. Principal metabolic pathways include superoxide dismutase (SOD), which is expressed as extracellular, intracellular and mitochondrial isoforms. These isoforms metabolize $\mathrm{O}_{2}{ }^{-}$to $\mathrm{H}_{2} \mathrm{O}_{2}$. Further metabolism by peroxidases, including catalase and glutathione peroxidase, yields $\mathrm{O}_{2}$ and $\mathrm{H}_{2} \mathrm{O}$ (7). Cells possess antioxidant systems to control the redox state, which is important for their survival. Excessive ROS production results in the activation of events that lead to death and survival in several types of cells (8-10). In addition, some evidence indicates that either ROS or the collapse of mitochondrial membrane potential $\left(\Delta \Psi_{\mathrm{m}}\right)$ opens the mitochondrial permeability transition pores. This occurrence is accompanied by the release of proapoptotic molecules such as cytochrome c into the cytoplasm (11), triggering apoptosis. Since AMA acts directly on the mitochondria, AMA-induced apoptosis has been reported in many experiments, including our recent report on HeLa and As4.1 juxtaglomerular cells (12-18).

The cell cycle in eukaryotes is regulated by cyclindependent kinases (CDKs). The cyclins, members of the cell cycle regulators, bind to and activate CDKs. The sequential formation, activation and subsequent inactivation of CDKs and cyclins is critical to the control of the cell cycle $(19,20)$. The cyclin-dependent kinase inhibitors (CDKIs) p21 and p27 also play key roles in controlling cell cycle progression by negatively regulating cyclin-dependent kinase (CDK) activity (19-21). Previously, it was noted that less traditional factors, as well as traditional cell cycle regulators such as nutrients, growth factors and hormones, may have an important impact on cell cycle regulation. Of the less traditional factors, ROS was commonly thought to be entirely toxic to cells, leading to cell death. Evidence shows that ROS may also act as a signaling molecule $(22,23)$ and, as such, may have a role in cell cycle progression.

We recently demonstrated that AMA produced ROS in HeLa cells and efficiently induced apoptosis, as evidenced by the flow cytometric detection of sub-G1 DNA content, annexin $\mathrm{V}$ binding assay and DAPI staining $(13,18)$. This apoptotic process was accompanied by the loss of mitochondrial membrane potential $\left(\Delta \Psi_{\mathrm{m}}\right)$, the down-regulation of $\mathrm{Bcl}-2$, the up-regulation of Bax, and the degradation of the PARP protein. In the present study, we investigated the antiapoptotic effects of $\mathrm{Z}-\mathrm{VAD}, \mathrm{SOD}$ or catalase in relation to the cell cycle in AMA-treated HeLa cells. 


\section{Materials and methods}

Cell culture. Human cervical adenocarcinoma HeLa cells were maintained in a humidified room atmosphere containing $5 \% \mathrm{CO}_{2}$ at $37^{\circ} \mathrm{C}$. HeLa cells were cultured in RPMI-1640 supplemented with $10 \%$ fetal bovine serum (FBS) and $1 \%$ penicillin-streptomycin (Gibco BRL, Grand Island, NY). Cells were routinely grown in 100-mm plastic tissue culture dishes (Nunc, Roskilde, Denmark) and harvested with a solution of trypsin-EDTA when they were in a logarithmic growth phase. Cells were maintained under culture conditions as described above for all experiments.

Reagents. AMA (Sigma-Aldrich Chemical Company, St. Louis, MO) was dissolved in ethanol at $2 \times 10^{-2} \mathrm{M}$ as a stock solution. Pan-caspase inhibitor (Z-VAD-FMK) was obtained from R\&D Systems Inc. (Minneapolis, MN) and dissolved in DMSO (Sigma) at $1 \times 10^{-2} \mathrm{M}$ as a stock solution, and was also used as a control vehicle. SOD and catalase were obtained from Sigma and dissolved in $50 \mathrm{mM}$ potassium phosphate buffer at $4733 \mathrm{U} / \mathrm{ml}$. The stock solutions were wrapped in foil and kept at $4^{\circ} \mathrm{C}$ or $-20^{\circ} \mathrm{C}$.

Growth inhibition assay. The in vitro growth inhibition effect of AMA with or without Z-VAD, SOD or catalase on HeLa cells was determined by measuring the 3-(4,5-dimethylthiazol2-yl)-2,5-diphenyltetrazolium bromide (MTT) dye absorbance of living cells as previously described (24). In brief, $2 \times 10^{5}$ cells/well were seeded onto 96-well microtiter plates (Nunc). After exposure to the indicated AMA concentration with or without Z-VAD, SOD or catalase for $72 \mathrm{~h}, 50 \mu \mathrm{l}$ of MTT solution (Sigma) (2 $\mathrm{mg} / \mathrm{ml}$ in PBS) were added to each well, and the plates were incubated for 3 or 4 additional hours at $37^{\circ} \mathrm{C}$. MTT solution in medium was withdrawn by pipetting. To achieve the solubilization of the formazan crystals that formed in viable cells, 100 or $200 \mu 1$ of DMSO were added to each well. The optical density of each well was measured at $570 \mathrm{~nm}$ using a microplate reader (Spectra MAX 340, Molecular Devices Co., Sunnyvale, CA). Each plate contained multiple wells of a given experimental condition and multiple control wells. This procedure was replicated in 2-4 plates/ condition.

Cell cycle and sub-G1 analysis. The distribution of the cell cycle and of sub-G1 was determined by staining DNA with propidium iodide (PI; Sigma-Aldrich), a fluorescent biomolecule that can be used to stain DNA, as previously described (25). In brief, $1 \times 10^{6}$ cells were incubated with $20 \mu \mathrm{M}$ AMA with or without Z-VAD, SOD or catalase for $72 \mathrm{~h}$. Cells were then washed with phosphate-buffered saline (PBS) and fixed in $70 \%$ ethanol. Cells were again washed with PBS and incubated with propidium iodine (PI; $10 \mu \mathrm{g} / \mathrm{ml}$ ) with the simultaneous treatment of RNase at $37^{\circ} \mathrm{C}$ for $30 \mathrm{~min}$. The percentage of cells in the different phases of the cell cycle or those with sub-G1 DNA content were measured with a FACStar flow cytometer (Becton-Dickinson, San Jose, CA) and analyzed using Lysis II and Cellfit software (Becton-Dickinson) or ModFit software (Verity Software House Inc., ME).

Detection of intracellular ROS. Intracellular ROS levels were detected by means of an oxidation-sensitive fluorescent probe

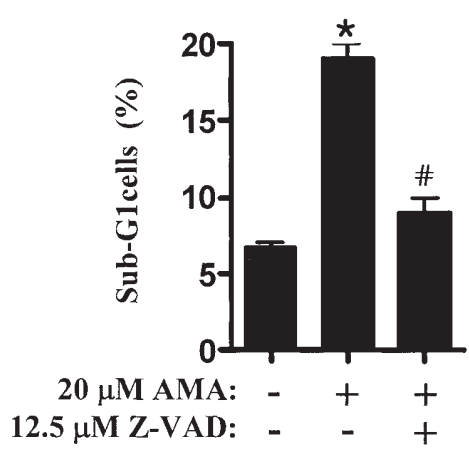

Figure 1. Effects of Z-VAD on cell death in AMA-treated HeLa cells. Exponentially growing cells were treated with the indicated AMA concentrations with or without Z-VAD $(12.5 \mu \mathrm{M})$ for $72 \mathrm{~h}$. The number of sub-G1 cells was assessed by DNA flow cytometric analysis. The percentage of sub-G1 cells is shown. ${ }^{*} \mathrm{P}<0.05$ compared to the HeLa control group. ${ }^{*} \mathrm{P}<0.05$ compared to cells treated with AMA alone.

dye, 2',7'-dichlorodihydrofluorescein diacetate ( $\left.\mathrm{H}_{2} \mathrm{DCFDA}\right)$ (Invitrogen Molecular Probes, Eugene, OR). $\mathrm{H}_{2}$ DCFDA was intracellularly deacetylated by non-specific esterase, which was further oxidized by cellular peroxides to the fluorescent compound, 2,7-dichlorofluorescein $(\mathrm{DCF})(\mathrm{Ex} / \mathrm{Em}=485 /$ $535 \mathrm{~nm}$ ). In brief, cells were incubated with AMA with or without Z-VAD, SOD or catalase for $72 \mathrm{~h}$. The cells were then washed in PBS and incubated with $20 \mu \mathrm{M} \mathrm{H}_{2}$ DCFDA at $37^{\circ} \mathrm{C}$ for $30 \mathrm{~min}$ according to the manufacturer's instructions. DCF fluorescence was detected using a FACStar flow cytometer (Becton-Dickinson). For each sample, 5,000 or 10,000 events were collected. ROS levels were expressed as the mean fluorescence intensity (MFI), which was calculated by CellQuest software.

Statistical analysis. Results represent the mean of two or three independent experiments; bar, SD. Microsoft Excel or Instat software (GraphPad Prism4, San Diego, CA) was used to analyze the data. The student's t-test or one-way analysis of variance (ANOVA) with post hoc analysis using Tukey's multiple comparison test was used for parametric data. Statistical significance was defined as $\mathrm{p}<0.05$.

\section{Results}

Effects of the pan-caspase inhibitor Z-VAD on apoptosis, cell growth and cell cycle distribution in AMA-treated HeLa cells. We examined the effects of the pan-caspase inhibitor Z-VAD on apoptosis, cell growth and cell cycle distribution in AMAtreated HeLa cells. As shown in Fig. 1, DNA flow cytometric analysis indicated that, at $72 \mathrm{~h}, 20 \mu \mathrm{M}$ of AMA significantly increased the number of sub-G1 cells compared with the control cells. Treatment with Z-VAD resulted in a marked rescue of HeLa cells from AMA-induced apoptosis in view of sub-G1 cells and annexin $\mathrm{V}$ exposure (data not shown). AMA also inhibited HeLa cell growth (Fig. 2A). Notably, treatment with Z-VAD did not significantly prevent the growth inhibition of HeLa cells by AMA (Fig. 2A). Treatment with AMA for $72 \mathrm{~h}$ induced S-phase arrest of the cell cycle in HeLa cells (Fig. 2B). The S-phase arrest by AMA was observed in a time (24-72 h)- and dose (0.5-50 $\mu \mathrm{M}$ )-dependent manner (data 
A

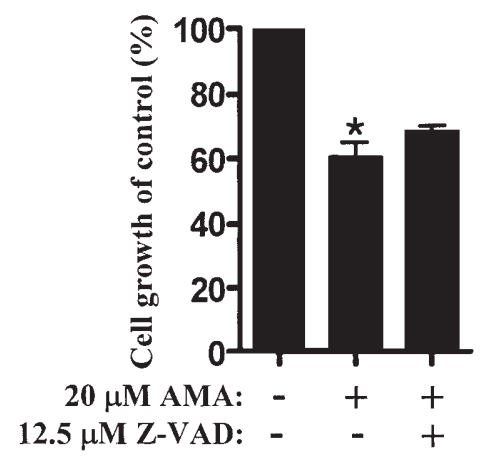

B

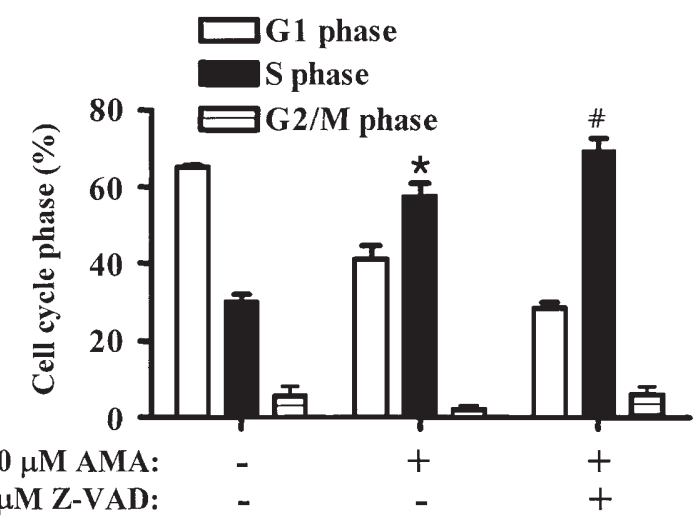

Figure 2. Effects of Z-VAD on cell growth and the cell cycle in AMA-treated HeLa cells. Exponentially growing cells were treated with the indicated AMA concentrations with or without Z-VAD $(12.5 \mu \mathrm{M})$ for $72 \mathrm{~h}$. (A) The growth of AMA- and/or Z-VAD-treated HeLa cells, assessed by MTT assay. (B) Cell cycle distribution in AMA- and/or Z-VAD-treated HeLa cells. * $\mathrm{P}<0.05$ compared to the HeLa control group. "P<0.05 compared to cells treated with AMA alone.

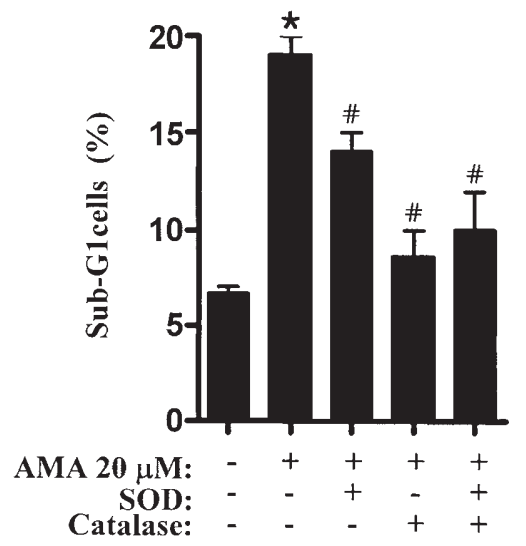

Figure 3. Effects of SOD and catalase on cell death in AMA-treated HeLa cells. Exponentially growing cells were treated with the indicated AMA concentrations with or without SOD and/or catalase for $72 \mathrm{~h}$. The number of sub-G1 cells was assessed by DNA flow cytometric analysis. The sub-G1 cell percentage is shown. ${ }^{*} \mathrm{P}<0.05$ compared to the HeLa control group. ${ }^{\#} \mathrm{P}<0.05$ compared to cells treated with AMA alone. not shown). In particular, following treatment with Z-VAD, $S$-phase arrest of the cell cycle in AMA-treated cells was significantly intensified (Fig. 2B). Treatment with Z-VAD $(12.5 \mu \mathrm{M})$ alone did not change cell growth inhibition and cell cycle distribution in control HeLa cells (data not shown).

Effects of exogenous SOD and catalase on apoptosis, cell growth and cell cycle distribution in AMA-treated HeLa cells. We examined whether exogenous SOD and catalase changed apoptosis and cell growth levels and cell cycle distribution in AMA-treated HeLa cells. As shown in Fig. 3, treatment with SOD $(30 \mathrm{U} / \mathrm{ml})$ and/or catalase $(30 \mathrm{U} / \mathrm{ml})$ significantly prevented apoptosis in AMA-treated HeLa cells at $72 \mathrm{~h}$ following the inspection of sub-G1 and annexin V-stained cells (data not shown). Treatment with SOD and/or catalase did not reduce the inhibition of growth in AMA-treated HeLa cells (Fig. 4A). In particular, S-phase arrest of the cell cycle in AMA-treated cells was enhanced by treatment with SOD and/or catalase (Fig. 4B). Neither synergistic nor additive effects of SOD
A

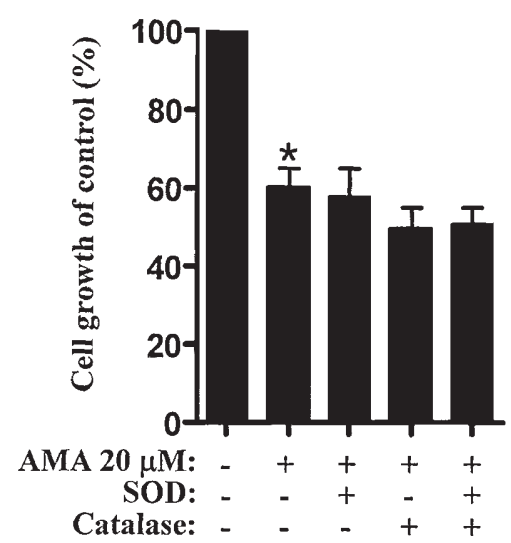

B

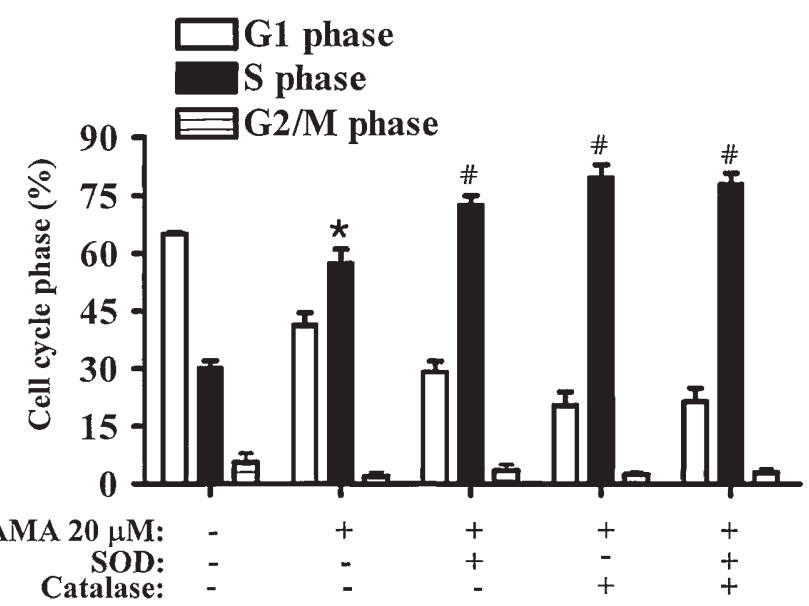

Figure 4. Effects of SOD and catalase on cell growth and the cell cycle in AMA-treated HeLa cells. Exponentially growing cells were treated with the indicated AMA concentrations with or without SOD and/or catalase for 72 h. (A) The growth of AMA- or SOD- and/or catalase-treated HeLa cells, which was assessed by MTT assay. (B) Cell cycle distribution in AMA- or SOD- and/or catalase-treated HeLa cells. * $<<0.05$ compared to the HeLa control group. ${ }^{\#} \mathrm{P}<0.05$ compared to cells treated with AMA alone. 


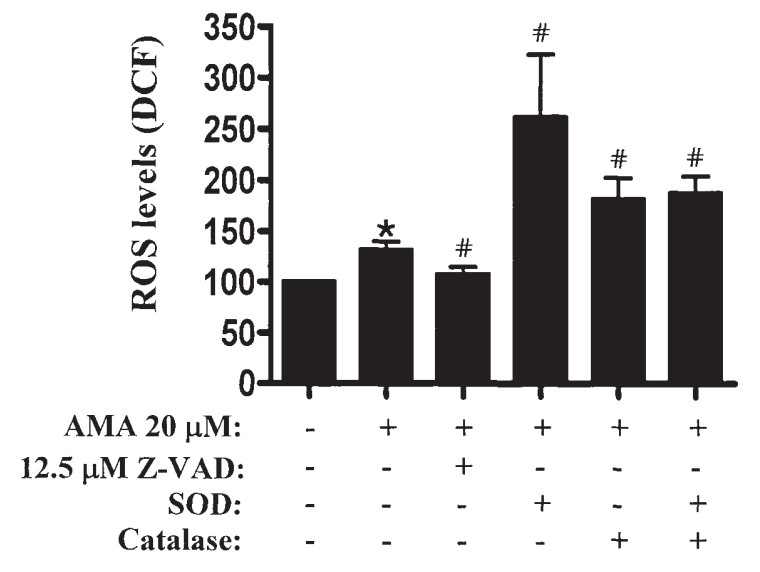

Figure 5. Effects of Z-VAD, SOD or catalase on ROS levels in AMA-treated HeLa cells. Exponentially growing cells were treated with the indicated AMA concentrations with or without Z-VAD, SOD and/or catalase for $72 \mathrm{~h}$. Intracellular ROS (DCF fluorescence) levels in HeLa cells were determined by a FACStar flow cytometer. ${ }^{*} \mathrm{P}<0.05$ compared to the HeLa control group. ${ }^{\#} \mathrm{P}<0.05$ compared to cells treated with AMA alone.

and catalase on the levels of apoptosis, growth inhibition and S-phase arrest in AMA-treated HeLa cells were observed (Figs. 3 and 4). Treatment with SOD and/or catalase alone did not significantly change cell growth inhibition and cell cycle distribution in control HeLa cells (data not shown).

Effects of Z-VAD, SOD and catalase on ROS levels in AMAtreated HeLa cells. To elucidate the involvement of ROS in cell cycle changes in HeLa cells, we assessed ROS levels using $\mathrm{H}_{2}$ DCFDA fluorescence. As shown in Fig. 5, ROS levels were increased in HeLa cells exposed to AMA for $72 \mathrm{~h}$. Z-VAD, which showed anti-apoptotic and enhanced S-phase arrest effects in AMA-treated HeLa cells, significantly reduced ROS levels. However, exogenous treatment with SOD and/or catalase showed a similar effect as Z-VAD, which increased ROS levels in AMA-treated HeLa cells. Neither synergistic nor additive effects of SOD and catalase on ROS levels in these cells were observed (Fig. 5). Treatment with Z-VAD, SOD or catalase alone did not significantly change ROS levels (data not shown).

\section{Discussion}

In the present study, we focused on evaluating the antiapoptotic effects of Z-VAD, SOD or catalase in relation to the cell cycle in AMA-treated HeLa cells, having demonstrated that AMA efficiently induces apoptosis (13). Cell cycle analysis has revealed that treatment with AMA significantly induces an S-phase arrest of the cell cycle for $72 \mathrm{~h}$. The S-phase checkpoint, termed the replication checkpoint, has previously been described (26-28). This checkpoint monitors S-phase progression and slows the rate of ongoing DNA synthesis. The S-phase checkpoint is thought to involve the activation of the ATM and ATR kinases, with the subsequent activation of Chk1 and Chk2 in response to DNA damage (26,29). Since AMA acts directly on the mitochondria and produces ROS in HeLa cells, it is possible that ROS readily damages biological molecules, especially DNA, inducing S-phase arrest of the cell cycle in HeLa cells. However, we recently reported that AMA did not induce any specific phase arrest of the cell cycle in As4.1 juxtaglomerular cells (12), and induced a G1-phase arrest in Calu-6 lung cancer cells (unpublished data). These results suggest that the specificity of cell cycle arrest by AMA depends on differences in cell type, and that AMA-induced ROS cannot always trigger the S-phase checkpoint, depending on the type of cell.

According to the current data, treatment with Z-VAD, SOD or catalase prevented AMA-induced apoptosis, but did not significantly reduce the inhibition of growth in AMAtreated HeLa cells. The anti-apoptotic effect of Z-VAD was associated with the down-regulation of ROS levels in AMAtreated HeLa cells, whereas that of SOD or catalase was correlated with the up-regulation of ROS levels in the cells. These results suggest that ROS changes by AMA, Z-VAD, SOD or catalase are not directly related to the induction of apoptosis. Interestingly, following treatment with Z-VAD, SOD or catalase, an intensified S-phase arrest of the cell cycle was observed in AMA-treated cells. The enhancement of S-phase arrest by these agents was not closely related to ROS levels. In AMA-treated HeLa cells, the S-phase arrest intensified by Z-VAD, SOD or catalase appeared to increase the resistance of cells to apoptosis. It is possible that the S-phase checkpoint prolonged/increased by Z-VAD, SOD or catalase in AMA-treated HeLa cells did not trigger the apoptotic events in the cells. However, the augmentation of S-phase arrest by these agents makes cells more resistant to apoptosis, and is in need of further study. We did not observe a synergistic or additive effect of SOD and catalase on cell death (sub-G1 cells), cell growth and S-phase arrest of the cell cycle. It is possible that catalase functions at the downstream region of SOD, and/or that SOD and catalase negatively regulate each other's entrance into cells.

In summary, we have demonstrated that AMA, an inhibitor of electron transport in mitochondria, induces the S-phase arrest of the cell cycle in HeLa cells. Treatment with Z-VAD, SOD or catalase decreased apoptotic levels in AMA-treated cells, which was accompanied by the enhancement of S-phase arrest of the cell cycle in these cells.

\section{Acknowledgements}

This study was supported by the Korean Science and Engineering Foundation (R01-2006-000-10544-0) and a Korea Research Foundation Grant funded by the Government of the Republic of Korea (MOEHRD).

\section{References}

1. Nakayama K, Okamoto F and Harada Y: Antimycin A: isolation from a new Streptomyces and activity against rice plant blast fungi. J Antibiot 9: 63-66, 1956.

2. Campo ML, Kinnally KW and Tedeschi H: The effect of antimycin A on mouse liver inner mitochondrial membrane channel activity. J Biol Chem 267: 8123-8127, 1992.

3. Maguire JJ, Kagan VE and Packer L: Electron transport between cytochrome $\mathrm{c}$ and alpha tocopherol. Biochem Biophys Res Commun 188: 190-197, 1992.

4. Pham NA, Robinson BH and Hedley DW: Simultaneous detection of mitochondrial respiratory chain activity and reactive oxygen in digitonin-permeabilized cells using flow cytometry. Cytometry 41: 245-251, 2000.

5. Balaban RS, Nemoto S and Finkel T: Mitochondria, oxidants, and aging. Cell 120: 483-495, 2005. 
6. Panduri V, Weitzman SA, Chandel NS and Kamp DW Mitochondrial-derived free radicals mediate asbestos-induced alveolar epithelial cell apoptosis. Am J Physiol Lung Cell Mol Physiol 286: L1220-L1227, 2004.

7. Wilcox CS: Reactive oxygen species: roles in blood pressure and kidney function. Curr Hypertens Rep 4: 160-166, 2002.

8. Dasmahapatra G, Rahmani M, Dent P and Grant S: The tyrphostin adaphostin interacts synergistically with proteasome inhibitors to induce apoptosis in human leukemia cells through a reactive oxygen species (ROS)-dependent mechanism. Blood 107 232-240, 2006.

9. Wallach-Dayan SB, Izbicki G, Cohen PY, Gerstl-Golan R, Fine A and Breuer R: Bleomycin initiates apoptosis of lung epithelial cells by ROS but not by Fas/FasL pathway. Am J Physiol Lung Cell Mol Physiol 290: L790-L796, 2006.

10. Simon HU, Haj-Yehia A and Levi-Schaffer F: Role of reactive oxygen species (ROS) in apoptosis induction. Apoptosis 5: 415-418, 2000

11. Petronilli V, Penzo D, Scorrano L, Bernardi P and Di Lisa F: The mitochondrial permeability transition, release of cytochrome $\mathrm{c}$ and cell death. Correlation with the duration of pore openings in situ. J Biol Chem 276: 12030-12034, 2001.

12. Han YW, Kim SZ, Kim SH and Park WH: The changes of intracellular $\mathrm{H} 2 \mathrm{O} 2$ are an important factor maintaining mitochondrial membrane potential of antimycin A-treated As4.1 juxtaglomerular cells. Biochem Pharmacol 73: 863-872, 2007.

13. Park WH, Han YW, Kim SH and Kim SZ: An ROS generator, antimycin A, inhibits the growth of HeLa cells via apoptosis. J Cell Biochem 102: 98-109, 2007.

14. Park WH, Han YW, Kim SW, Kim SH, Cho KW and Kim SZ: Antimycin A induces apoptosis in As4.1 juxtaglomerular cells. Cancer Lett 251: 68-77, 2007.

15. King MA: Antimycin A-induced killing of HL-60 cells: apoptosis initiated from within mitochondria does not necessarily proceed via caspase 9. Cytometry A 63: 69-76, 2005.

16. Kaushal GP, Ueda N and Shah SV: Role of caspases (ICE/CED 3 proteases) in DNA damage and cell death in response to a mitochondrial inhibitor, antimycin A. Kidney Int 52: 438-445, 1997.
17. De Graaf AO, Meijerink JP, van den Heuvel LP, et al: Bcl-2 protects against apoptosis induced by antimycin $\mathrm{A}$ and bongkrekic acid without restoring cellular ATP levels. Biochim Biophys Acta 1554: 57-65, 2002.

18. Han YH, Kim SH, Kim SZ and Park WH: Intracellular GSH levels rather than ROS levels are tightly related to AMAinduced HeLa cell death. Chem Biol Interact 171: 67-78, 2008.

19. Kastan MB and Bartek J: Cell-cycle checkpoints and cancer. Nature 432: 316-323, 2004

20. Massague J: G1 cell-cycle control and cancer. Nature 432: 298-306, 2004.

21. Coqueret O: New roles for p21 and p27 cell-cycle inhibitors: a function for each cell compartment? Trends Cell Biol 13: 65-70, 2003.

22. Shackelford RE, Kaufmann WK and Paules RS: Oxidative stress and cell cycle checkpoint function. Free Radic Biol Med 28: 1387-1404, 2000

23. Boonstra J and Post JA: Molecular events associated with reactive oxygen species and cell cycle progression in mammalian cells. Gene 337: 1-13, 2004.

24. Park WH, Seol JG, Kim ES, et al: Arsenic trioxide-mediated growth inhibition in MC/CAR myeloma cells via cell cycle arrest in association with induction of cyclin-dependent kinase inhibitor, p21, and apoptosis. Cancer Res 60: 3065-3071, 2000.

25. Park WH, Jung CW, Park JO, et al: Trichostatin inhibits the growth of ACHN renal cell carcinoma cells via cell cycle arrest in association with p27, or apoptosis. Int J Oncol 22: 1129-1134, 2003.

26. Zhou BB and Elledge SJ: The DNA damage response: putting checkpoints in perspective. Nature 408: 433-439, 2000.

27. Shapiro GI: Cyclin-dependent kinase pathways as targets for cancer treatment. J Clin Oncol 24: 1770-1783, 2006.

28. Schwartz GK and Shah MA: Targeting the cell cycle: a new approach to cancer therapy. J Clin Oncol 23: 9408-9421, 2005.

29. Falck J, Mailand N, Syljuasen RG, Bartek J and Lukas J: The ATM-Chk2-Cdc25 A checkpoint pathway guards against radioresistant DNA synthesis. Nature 410: 842-847, 2001. 